\title{
Heterozygotes for $H F E$ mutations have no increased risk of advanced alcoholic liver disease
}

\author{
J Grove, A K Daly, A D Burt, M Guzail, O F W James, M F Bassendine, C P Day
}

\begin{abstract}
Background-Iron overload is common in the livers of alcoholics and may play a role in disease pathogenesis. An MHC like gene, $H F E$, has recently been identified that is mutated in most patients with hereditary haemochromatosis (C282Y in $90 \%$ and $\mathrm{H} 63 \mathrm{D}$ in $45 \%$ of the remainder). Aim-To examine the hypothesis that these mutations determine hepatic iron status in alcoholics and play a role in predisposition to advanced alcoholic liver disease.

Methods-The HFE gene was genotyped in 257 patients with alcoholic liver disease and 117 locally matched healthy volunteers. In addition, iron staining was scored (0-4) on biopsy specimens from fibroticl cirrhotic patients with and without $H F E$ mutations matched for age and sex.

Results-Some $15.7 \%$ of fibrotic/cirrhotic patients were $\mathrm{C} 282 \mathrm{Y}$ heterozygotes compared with $13.7 \%$ of controls $(p=0.77)$. One control and three patients were C282Y homozygotes. Of chromosomes without the C282Y mutation, 68/442 (15.4\%) of patients' chromosomes carried the H63D mutation compared with $36 / 216$ $(\mathbf{1 6 . 6 \% )}$ of control chromosomes ( $p=$ 0.91). Significant (>grade 1) hepatocyte iron staining was seen in $6 / 23 \mathrm{C} 282 \mathrm{Y}$ heterozygotes and 4/26 H63D heterozygotes compared with $4 / 23$ controls.

Conclusions-Possession of a single copy of either of the two $H F E$ mutations influences neither liver iron content nor the risk of fibrotic disease in alcoholics. (Gut 1998;43:262-266)
\end{abstract}

Centre for Liver Research, University of Newcastle upon Tyne, UK

J Grove

A D Burt

M Guzail

O F W James

M F Bassendine

C P Day

Department of

Pharmacological

Sciences

J Grove

A K Daly

Correspondence to: Dr C P Day, Department of Medicine, The Medical School, Framlington Place, Newcastle upon Tyne NE2 $4 \mathrm{HH}, \mathrm{UK}$.

Accepted for publication 18 February 1998 rium. Studies from the United States, Australia, ${ }^{4}$ France, ${ }^{5}$ and the United Kingdom ${ }^{6}$ have shown that more than $85 \%$ of patients with a previous clinical diagnosis of $\mathrm{HHC}$ are homozygous for the C282Y mutation. A further $5 \%$ are heterozygous for this mutation, and, of these, $86 \%$ are also heterozygous for the H63D mutation. Of the remaining $10 \%$, almost half possess at least one copy of the H63D mutant allele. This compares with a heterozygote frequency in controls of $11 \%$ for the C282Y mutation and $25 \%$ for the H63D mutation.

It has been previously suggested that the haemochromatosis gene may not be completely recessive, with heterozygotes being at increased risk of other diseases in which iron may play a pathogenic role, including porphyria cutanea tarda, diabetes, certain malignancies, and coronary artery disease. ${ }^{7}$ The identification of the HFE gene has now enabled the study of these potential associations which, until now, has been hampered by the difficulty of accurately identifying the heterozygous state. Already a strong association has been shown between the possession of one or two copies of the $\mathrm{C} 282 \mathrm{Y}$ mutation and porphyria cutanea tarda, with a relative risk for disease of 6.2 (95\% confidence interval (CI) $2.6-14.5) .{ }^{8}$ Evidence from both animal models ${ }^{910}$ and humans ${ }^{11}{ }^{12}$ suggests that iron plays an important role in the pathogenesis of alcoholic liver disease (ALD). The mechanism of this apparent synergy between alcohol and iron is considered to be promotion of oxidative stress by iron with subsequent increased lipid peroxidation, a critical pathogenic mechanism in both HHC and ALD. ${ }^{13}$

It is well established that only a minority of heavy drinkers, estimated at between 10 and $20 \%$, will ever develop advanced ALD, ${ }^{14}$ and there is no doubt that the risk of disease increases with cumulative alcohol intake. ${ }^{15}$ Twin studies suggest that there is also a genetic component to disease susceptibility, ${ }^{16}$ and in white populations significant associations have recently been reported between ALD risk and polymorphisms of the genes encoding the cytochrome $P-450 \quad \mathrm{CYP} 2 \mathrm{E} 1^{17}$ and tumour necrosis factor $\alpha .^{18}$ In view of the evidence supporting a role for iron in the pathogenesis of ALD and the not infrequent finding of significant iron deposition in the livers of alcoholics, ${ }^{19}$ we have examined the hypothesis that these patients may be heterozygous for $H F E$ mutations, with the subsequent iron overload increasing their susceptibility to advanced ALD.

\section{Patients and methods}

SUBJECTS

After obtaining informed consent, blood (10 $\mathrm{ml})$ was collected from all consecutive unrelated patients with suspected or known ALD 
attending the liver unit from 1993. Patients were recruited from the clinic and liver ward. All were white and originated in the north east of England, as did their parents and grandparents. They had all consumed more than 8 units (about $64 \mathrm{~g}$ ethanol) per day for 10 or more years and had increased alanine aminotransferase, alkaline phosphatase or bilirubin on at least three occasions within a six month period. The history of alcohol consumption was verified by family members or friends when possible. Patients were excluded if their biopsy specimens suggested another cause for their liver disease or if they had serological evidence of previous hepatitis $B$ virus or hepatitis $C$ virus infection (second generation enzyme linked immunosorbent assay (ELISA)) or autoimmune liver disease. Healthy control subjects were randomly recruited from hospital and university staff. The only selection criterion was local origin.

HISTOLOGY

The diagnosis of ALD was confirmed by liver biopsy in all patients except when contraindicated by coagulopathy. Before biopsy all patients had a liver ultrasound examination to exclude biliary obstruction. Biopsy samples were categorised according to the presence or absence of (a) cirrhosis, (b) precirrhotic perivenular/pericellular fibrosis, and (c) steatohepatitis, defined as the presence of steatosis, ballooning degeneration, and Mallory's hyaline with or without neutrophil satellitosis. In addition, liver biopsy specimens were scored for iron deposition in all patients possessing one or two copies of either mutant allele and those patients with fibrosis/cirrhosis with neither mutation who were "matched" for age and sex to the $\mathrm{C} 282 \mathrm{Y}$ heterozygotes. Iron content was determined on sections stained with Perls' stain using the method of Searle et al. ${ }^{20}$ The presence of perisinusoidal and periportal tract iron was noted and the severity of hepatocyte iron staining was graded from 1 to 4 by two observers (MG and $\mathrm{ADB}$ ) unaware of $H F E$ genotype. In the absence of a histological examination of the liver, only patients with definite features of decompensation (varices, ascites, or encephalopathy) were categorised as cirrhotic. No attempt was made to diagnose precirrhotic fibrosis or steatohepatitis without liver biopsy. Age at presentation was considered either as the age at index biopsy or, when biopsy was contraindicated, as the age at the onset of decompensation. ${ }^{17}$

DETERMINATION OF HFE GENOTYPE

To detect the C282Y and H63D mutations, genomic DNA extracted from whole blood was amplified by polymerase chain reaction (PCR). ${ }^{2}$ The C282Y mutation creates a new RsaI restriction site and the H63D mutation abolishes a $B c l$ I site allowing identification by restriction enzyme digestion.

STATISTICAL ANALYSIS

Differences between genotypes and allele frequencies and deviations from the HardyWeinberg equilibrium were analysed using the $\chi^{2}$ test or Fisher's exact test depending on the minimum expected values. Differences in age at presentation between patients with different genotypes were compared using analysis of variance or unpaired $t$ tests.

\section{Results}

HISTOLOGY

Liver biopsy was performed in 221/257 patients; 162 had established cirrhosis and 43 precirrhotic fibrosis. Co-existing alcoholic steatohepatitis was present in 71 of the cirrhotics and 23 of those with precirrhotic fibrosis. One patient had steatohepatitis with no evidence of fibrosis and 15 had only simple steatosis. The remaining 36 patients were classified as cirrhotic on the basis of clinical evidence of severe portal hypertension (oesophageal varices or ascites) together with a prolonged prothrombin time precluding liver biopsy. There was no difference in age at biopsy or presentation between patients with and without cirrhosis $(48.7(0.76) v 48.55(1.20))$ or with and without steatohepatitis $(47.7$ (1.03) $v 49.4(0.89))$ (mean (SD)).

\section{GENOTYPING}

Table 1 shows the distribution of the two $H F E$ mutations among the healthy controls and the patients with different histological categories of ALD. The distribution of genotypes in all groups was in Hardy-Weinberg equilibrium. Some $15.7 \%$ of patients $(15.9 \%$ men, $14.7 \%$ women) with fibrosis/cirrhosis were heterozygous for the C282Y mutation compared with $19.9 \%$ of patients with fatty liver alone and $13.7 \%$ of controls $(p=0.77)$. Two patients with fibrosis/cirrhosis, one with fatty liver, and one control were homozygous for the mutation giving an allele frequency of 0.087 in fibrotic patients, 0.167 in patients with fatty liver only, and 0.077 in controls $(\mathrm{p}=0.26)$. Of chromosomes without the C282Y mutation (and therefore "at risk" of possessing the H63D mutation), 68/442 (15.4\%) from patients with fibrotic ALD carried the H63D mutation, compared with $4 / 25(16.0 \%)$ from patients with fatty liver and 36/216 (16.6\%) from controls $(p=0.91)$. Seven $(2.9 \%)$ patients with fibrotic ALD and one $(0.85 \%)$ control were homozygous for the H63D mutation and identical numbers were compound heterozygotes. One patient with fatty liver was also a compound heterozygote. Categorising the patients according to the presence of steatohepatitis disclosed no difference in the frequency of either mutation between patients with or without this lesion or between patients with hepatitis and controls (table 1).

To determine whether possession of $H F E$ mutations might lead to fibrotic alcoholic liver disease developing at an earlier stage in excessive alcohol drinkers, the age of presentation was compared in patients with fibrosis/cirrhosis according to genotype. We have shown previously that the age of patients with ALD correlates with cumulative lifetime alcohol intake. ${ }^{17}$ There was no difference between patients with and without a single copy of the C282Y mutation (46.8 (1.5) $v 49$ (0.72); $\mathrm{p}=0.21)$ or with 


\begin{tabular}{lccccc}
\hline \multicolumn{7}{l}{ Number of cases (\%) } \\
\cline { 2 - 6 } HFE genotype & Fibrotic ALD & Alcoholic hepatitis & No hepatitis & Fatty liver & Controls \\
\hline Wild type homozygote & $148(61.1)$ & $58(61.1)$ & $69(60.0)$ & $8(53.3)$ & $66(56.4)$ \\
H63D heterozygote & $47(19.4)$ & $20(21.1)$ & $23(20.0)$ & $3(20)$ & $33(28.2)$ \\
H63D homozygote & $7(2.9)$ & $3(3.1)$ & $3(2.6)$ & - & $1(0.85)$ \\
C282Y heterozygote & $31(12.8)$ & $11(11.5)$ & $14(12.1)$ & $2(13.3)$ & $15(12.8)$ \\
C282Y, H63D heterozygote & $7(2.9)$ & $2(2.1)$ & $5(4.3)$ & $1(6.6)$ & $1(0.85)$ \\
C282Y homozygote & $2(0.83)$ & $1(1.1)$ & $1(0.9)$ & $1(6.6)$ & $1(0.85)$ \\
Total & $242(100)$ & $95(100)$ & $115(100)$ & $15(100)$ & $117(100)$ \\
\hline
\end{tabular}

Fibrotic ALD includes patients with cirrhosis and precirrhotic fibrosis.

and without a single copy of the H63D mutation (49.6 (1.3) $v 48.4$ (0.77); $\mathrm{p}=0.44)$. Neither the seven H63D homozygotes (46.9 (2.5)) nor the compound heterozygotes (49.6 (5.5)) with fibrosis/cirrhosis were significantly different in age of presentation from the wild type patients $(48.9(0.89))$.

HEPATIC IRON CONTENT AND GENOTYPE

Table 2 shows the results of liver biopsy iron "scoring". Of the simple heterozygotes, 6/23 patients with the C282Y mutation and $4 / 26$ with the H63D mutation had significant hepatocyte iron deposition (>grade 1), compared with $4 / 23$ with neither mutation $(p=0.61)$. A further three C282Y heterozygotes and three H63D heterozygotes had grade 1 iron staining. Four of the five compound heterozygotes in whom a liver biopsy was available had grade 2 or 3 iron staining. Of the three patients homozygous for the C282Y mutation, two had grade 3 iron staining and the other grade 1 staining only. No cases showed grade 4 staining. In all but $1 / 29$ biopsy samples hepatocyte iron staining was associated with both perisinusoidal and portal tract (mesenchymal) iron staining. There was no correlation between recent alcohol consumption and iron staining. Of the patients with hepatocyte iron staining $21 / 29(72 \%)$ were active drinkers before biopsy compared with $42 / 57$ (74\%) of patients without iron staining.

\section{Discussion}

The hypothesis that we set out to examine in this study that heterozygotes for mutations in $H F E$ might be at increased risk of developing fibrotic ALD appeared well founded. Synergy between alcohol and HHC heterozygosity is suggested by a recent study of 1058 HHC heterozygotes, 39 of whom underwent liver biopsy. Four of the six biopsy specimens with fibrosis were from heavy alcohol misusers. ${ }^{21}$ Our results, however, provide strong evidence against simple heterozygotes for either mutation having an increased risk of advanced ALD. Compared with local controls, there was no increase in the frequency of either HFE mutation in alcoholic patients with fibrosis/ cirrhosis or steatohepatitis. The frequency of the H63D mutation in "at risk" control chromosomes $(16.6 \%)$ was identical with that observed in other white populations $(17.0 \%),{ }^{6}$ while the C282Y mutation frequency $(7.7 \%)$ was slightly higher than in previous British $(5.9 \%)^{68}$ and American $(3.2 \%)^{3}$ studies. This ethnic variation may account for the positive association between C282Y and ALD recently reported in preliminary form by a group from London, where one might expect ethnic origin to be more diverse than in north east England. ${ }^{22}$ Our results agree with those from a previous study that determined HLA antigens in patients with HHC and ALD associated with iron overload. ${ }^{23}$

The explanation for the lack of any association between HFE mutations and ALD is suggested by the results of iron staining. Formal quantification of liver iron, although ideal, was largely precluded by the retrospective nature of the study. However, since siderosis of grade 2 or above is usually associated with increased liver iron concentration, ${ }^{24}$ we can deduce from the iron staining results that patients with fibrotic ALD and a single copy of either mutation are no more likely to have significant hepatocyte iron accumulation than patients without the mutations. Simple heterozygotes therefore are not more susceptible to developing iron catalysed oxidative stress during ethanol consumption and accordingly have no increased risk of hepatic necroinflammation or fibrosis. The increased numbers of patients with grade 1 iron deposition (table 2) observed in the C282Y heterozygotes is consistent with results from previous studies of HHC heterozygotes. ${ }^{21}{ }^{25}$ The results of $H F E$ genotyping would, however, suggest that this mild increase in liver iron is not sufficient to increase the risk of fibrosis/cirrhosis in a heavy drinker.

Table 2 Hepatic iron staining in patients with fibrotic alcoholic liver disease and different HFE genotypes

\begin{tabular}{|c|c|c|c|c|c|}
\hline \multirow[b]{2}{*}{ HFE genotype } & \multicolumn{4}{|c|}{ Number with hepatocyte iron grade (\%) } & \multirow[b]{2}{*}{ Total } \\
\hline & 0 & 1 & 2 & 3 & \\
\hline Wild type homozygote & $19(83)$ & $0(0)$ & $3(13)$ & $1(4)$ & $23(100)$ \\
\hline H63D heterozygote & $19(73)$ & $3(12)$ & $3(12)$ & $1(4)$ & $26(100)$ \\
\hline H63D homozygote & $4(67)$ & $0(0)$ & $2(33)$ & $0(0)$ & $6(100)$ \\
\hline $\mathrm{C} 282 \mathrm{Y}$ heterozygote & $14(61)$ & $3(13)$ & $4(17)$ & $2(9)$ & $23(100)$ \\
\hline C282Y, H63D heterozygote & $1(20)$ & $0(0)$ & $2(40)$ & $2(40)$ & $5(100)$ \\
\hline C282Y homozygote & $0(0)$ & $1(33)$ & $0(0)$ & $2(67)$ & $3(100)$ \\
\hline
\end{tabular}

Wild type homozygotes were selected to match the C282Y heterozygotes for sex and age ( \pm 3 years). Grade 1 iron, barely discernible at low power magnitude; grade 2 , discrete granules seen at medium power; grade 3 , discrete granules seen at low power; grade 4 , masses visible at low power. 
Our results suggest that, in certain individuals, heavy alcohol consumption can lead to excessive accumulation of liver iron independent of $H F E$ genotype. Since it seems likely that the iron may be playing a role in disease pathogenesis in these individuals, it is important to consider possible alternative explanations for alcohol associated siderosis. Our observation that liver iron staining is not influenced by recent alcohol history is consistent with a previous study that showed no correlation between hepatic iron content and alcohol history, ${ }^{24}$ and would appear to argue against a direct effect of alcohol on liver iron. There is some evidence that the folic acid deficiency associated with chronic alcohol intake may increase iron absorption $^{26}$; however, a more intriguing explanation is suggested by the results from recent studies on the cellular mechanism of iron regulation. ${ }^{27}$ Like iron deficiency, oxidative stress increases the activity of iron regulatory proteins which bind to iron response elements in the mRNAs of iron regulating genes such as the transferrin receptor and ferritin. This results in an increase in the concentration of free iron within the cell. In ALD, therefore, the excessive iron deposition may be an effect as well as a cause of oxidative stress. Hepatic siderosis in an alcoholic may identify those patients who, for environmental and/or genetic reasons, are most susceptible to oxidative stress. In support, a recent study has shown an inverse correlation between liver iron staining and survival in patients with alcoholic cirrhosis. $^{28}$

The results presented do not allow any conclusions to be drawn about either the compound heterozygous state or H63D homozygosity and risk of ALD. Although both genotypes were present in a higher proportion of patients than controls $(2.9 \% v 0.85 \%)$, this was only slightly higher than the proportions expected from the control frequencies of each mutation (expected frequency of both genotypes $2.4 \%$ ). It may be expected that compound heterozygotes would be at increased risk of ALD since this genotype is thought to have low penetrance for $\mathrm{HHC}^{2}$ and may be phenotypically expressed by alcohol. The presence of significant iron staining in four of five biopsy specimens with this genotype would support this hypothesis; however, the low frequency of the compound heterozygous state means that it is unlikely to contribute significantly to the population risk of ALD.

Of the three patients (all male) homozygous for the C282Y mutation, in two HHC was probably exacerbated by excessive alcohol intake since they had grade 3 hepatocyte iron staining and histological results compatible with HHC with some additional alcohol related features (steatohepatitis and perivenular fibrosis). The third patient presented aged 45 with an alcohol intake of $600 \mathrm{~g}$ per week. Liver biopsy showed steatosis and grade 1 hepatocyte iron staining. The hepatic iron index was 0.15 (normal range $<1.5$ ) and liver iron concentration was $7 \mu \mathrm{mol} / \mathrm{g}$ dry weight (normal range $<33 \mu \mathrm{mol} / \mathrm{g}$ ). The low iron index despite his heavy alcohol consumption raises doubt as to whether he will ever develop significant hepatic iron deposition or other features of the HHC phenotype. This lack of universal phenotypic expression of C282Y homozygosity is supported by a recent study of patients with porphyria cutanea tarda in which 86 year olds were identified with this genotype but with no clinical evidence of HHC. ${ }^{8}$

In conclusion, we have demonstrated that heterozygotes for either of the recently described mutations in the $H F E$ gene are not at increased risk of ALD since possession of a single copy of these mutations does not significantly increase liver iron content above that seen in a proportion of patients with ALD. We have also provided evidence that the genotype most closely associated with $\mathrm{HHC}$ is not always phenotypically expressed even in the presence of excessive alcohol intake. From a practical point of view it would appear pertinent to determine $H F E$ genotype in patients with suspected clinical ALD who have significant hepatocyte iron staining, although the common HHC genotype (C282Y homozygote) will be found in less than one in 10 cases. Future studies should be directed at elucidating factors that determine the phenotypic expression of $H F E$ mutations and liver iron content in ALD, and prospective studies aimed at determining the pathological and prognostic significance of iron deposition associated with excessive alcohol intake.

We thank Sister Helen Milburn for invaluable assistance with sample collection and logging. This work was supported by a Medical Research Council Project Grant.

1 Dadone MM, Kushner JP, Edwards CQ, et al. Hereditary hemochromatosis: analysis of laboratory expression of the disease by genotype in 18 pedigrees. Am f Clin Pathol 1982;78:196-207.

2 Feder JN, Gnirke A, Thomas W, et al. A novel MHC class I-like gene is mutated in patients with hereditary hemochromatosis. Nat Genet 1996;13:399-408.

3 Calandro L, Thorsen T, Barcellos L, et al. Mutation analysis in hereditary hemochromatosis. Blood Cells Mol Dis 1996;22:194A-94B.

4 Jazwinska EC, Cullen LM, Busfield F, et al. Haemochromatosis and HLA-H. Nat Genet 1996;14:249-51.

5 Jouanolle AM, Gandon G, Jezequel P, et al. Haemochromatosis and HLA-H. Nat Genet 1996;14:251-2.

6 The UK Haemochromatosis Consortium. A simple genetic test identifies $90 \%$ of UK patients with haemochromatosis. Gut 1997;41:841-4

7 Beutler E. Genetic irony beyond hemochromatosis: clinical effects of HLA-H mutations. Lancet 1997;349:296-7.

8 Roberts AG, Whatley SD, Morgan RR, et al. Increased frequency of the hemochromatosis Cys282Tyr mutation in sporadic porphyria cutanea tarda. Lancet 1997;349:321-3.

9 Tsukamoto H, Horne W, Kamimura S, et al. Experimental liver cirrhosis induced by alcohol and iron. 7 Clin Invest 1995;96:620-30

10 Stal P, Hultcranz R. Iron increases ethanol toxicity in rat liver. F Hepatol 1993;17:108-15.

11 Adams PC, Agnew S. Alcoholism in hereditary hemochromatosis revisited: prevalence and clinical consequences among homozygous siblings. Hepatology 1996;23:724-7.

12 Le Sage GD, Baldus WB, Fairbanks VF, et al. Hemochromatosis: genetic or alcohol-induced? Gastroenterology 1983;84:1471-7.

13 Day CP. Is necroinflammation a prerequisite for fibrogenesis? Hepato-Gastroenterology 1996;43:104-20.

14 Day CP, Bassendine MF. Genetic predisposition to alcoholic liver disease. Gut 1992;33:1444-7.

15 Lelbach WK. Cirrhosis in the alcoholic and its relation to the volume of alcohol abuse. Ann NY Acad Sci 1975;252: 85-105.

16 Hrubec Z, Omenn GS. Evidence of a genetic predisposition to alcoholic cirrhosis and psychosis; twin concordances for alcoholism and its biological end points by zygosity among male veterans. Alcohol Clin Exp Res 1981;9:306-9.

17 Grove J, Daly AK, Brown AstJM, et al. The RsaI polymorphism of CYP2E1 and susceptibility to alcoholic iver disease in Caucasians: effect on age of presentation and dependence on alcoh

18 Grove J, Daly AK, Bassendine MF, et al. Association of a tumor necrosis factor polymorphism with susceptibility to alcoholic steatohepatitis. Hepatology 1997;26:143-6. 
19 Ludwig J, Hashimoto E, Porayko MK, et al. Hemosiderosis in cirrhosis: a study of 447 native livers. Gastroenterology in cirrhosis: a st

20 Searle J, Kerr JFR, Halliday JW, et al. Iron storage disease. In: MacSween RNM, Anthony PP, Scheuer PJ et al, eds. Pathology of the liver. 3rd edn. New York: Churchill Living-

21 Bulaj ZL, Griffen LM, Jorde LB, et al. Clinical and biochemical abnormalities in people heterozygous for hemochromatosis. N Eng f Med 1996;335:1799-805.

22 Teare J, Mantafounis D, Yallop R, et al. Increased frequency of the haemochromatosis allele in patients with alcoholic liver disease. $\mathcal{F}$ Hepatol 1997;26(suppl 1):57.

23 Simon $\mathrm{M}$, Bourel $\mathrm{M}$, Genetet B, et al. Idiopathic hemochromatosis and iron overload in alcoholic liver disease: differentiation by HIA phenotype. Gastroenterology 1977;73:655-8.
24 Chapman RW, Morgan MY, Laulicht M, et al. Hepatic iron stores and markers of iron overload in alcoholics and patients with idiopathic hemochromatosis. Dig Dis Sci

25 Bassett ML, Halliday JW, Powell LW. Value of hepatic iron measurements in early hemochromatosis and determination of the critical iron level associated with fibrosis. Hepatology 1986;6:24-9.

26 MacDonald RA, Jones RS, Pechet GS. Folic acid deficiency and hemochromatosis. Arch Pathol 1965;80:153-60.

27 Rouault TA, Klausner RD. Iron-sulfur clusters as biosensors of oxidants and iron. Trends Biochem Sci 1996;21:174-7.

28 Ganne-Carrie N, Christidis C, Chevret S, et al. Predictive value of liver iron content for survival in 229 prospectively followed patients with cirrhosis. $\mathcal{7}$ Hepatol 1996;25 (supp 1):95. 